\title{
Trajectories of Fetal Adipose Tissue Thickness in Pregnancies After Gastric Bypass Surgery
}

\author{
Gülen Yerlikaya-Schatten ${ }^{1} \cdot$ Michael Feichtinger ${ }^{1,2} \cdot$ Tina Stopp $^{1} \cdot$ Evelyn A. Huhn ${ }^{3} \cdot$ Kinga Chalubinski $^{1}$. \\ Peter Husslein ${ }^{1} \cdot$ Wolfgang Eppel $^{1} \cdot$ Christian Schatten $^{1} \cdot$ Christian S. Göbl $^{1}$ (D)
}

Published online: 14 August 2019

(C) The Author(s) 2019

\begin{abstract}
Purpose Recent studies showed that women after surgery are at higher risk of delivering small-for-gestational infants. Thus, this study aims to investigate longitudinal changes of fetal subcutaneous adipose tissue thickness (FSCTT) of fetuses conceived after gastric bypass surgery as compared to BMI-matched controls.

Methods Retrospective cohort study measuring ultrasound-derived longitudinal trajectories of abdominal FSCTT in 41 singleton pregnancies after gastric bypass surgery compared to 41 BMI-matched controls and 64 obese mothers.

Results FSCTT was significantly lower in fetuses of women after GB as compared to BMI-matched controls in the second (mean difference $1.38 \mathrm{~mm}, p<0.001$ ) and third trimester of gestation (mean difference $3.37 \mathrm{~mm}, \mathrm{p}<0.001$ ). Longitudinal analysis revealed significant differences in mean FSCTT trajectories between offspring's in GB mothers, BMI-matched, or obese controls. The ratio of FSCTT and abdominal circumference remained constant in the BMI-matched control group whereas it significantly decreased in fetuses of women after GB. Despite remarkable differences were observed in longitudinally assessed FSCTT, further analyses in the GB subgroup revealed that FSCTT were not influenced by OGTT mean or 120 min glucose values, biochemically hypoglycemia, time since bariatric surgery, or weight loss since surgery.

Conclusion In fetuses of mothers with history of bariatric surgery, abdominal FSCTT was markedly reduced. While the underlying mechanisms are not fully understood, a multifactorial genesis including nutritional deficiencies and altered metabolism after bariatric surgery is assumed.
\end{abstract}

Keywords Bariatric surgery $\cdot$ Fetus $\cdot$ Growth restriction $\cdot$ Fat tissue $\cdot$ Gestational diabetes $\cdot$ OGTT

\section{Introduction}

The number of obese women in childbearing age undergoing bariatric surgery has risen significantly within the past years [1]. Although bariatric surgery is the most effective treatment

Electronic supplementary material The online version of this article (https://doi.org/10.1007/s11695-019-04115-9) contains supplementary material, which is available to authorized users.

Christian S. Göbl

christian.goebl@meduniwien.ac.at

1 Department of Obstetrics and Gynaecology, Division of Obstetrics and Feto-Maternal Medicine, Medical University of Vienna, Waehringer Guertel 18-20, A-1090 Vienna, Austria

2 Wunschbaby Institut Feichtinger, Vienna, Austria

3 Department of Obstetrics and Gynaecology, University Hospital Basel, Basel, Switzerland for morbid obesity in diabetic patients [2,3], recent studies showed that women after surgery are at higher risk of perinatal complications such as preterm birth or delivering small-forgestational (SGA) infants [4-7]. In this context, we recently demonstrated a continuous decline in fetal growth throughout pregnancy after gastric bypass surgery, starting at the early second trimester [8]. Although the pathophysiologic mechanism beyond this observation remains unclear, it is believed that fetal growth delay is rather caused by nutrient deficiency and postprandial hypoglycemia (due to dumping syndrome) than by growth restriction due to placenta etiology or preeclampsia [5,9].

Previous studies indicated that trajectories of ultrasoundderived fetal subcutaneous adipose tissue thickness (FSCTT) are very sensitive to maternal metabolic alterations during pregnancy like gestational diabetes (GDM) [10] and closely related to established predictors of fetal nutrition status such as amniotic fluid, abdominal circumference, ponderal index, and 
triceps and subscapular skinfold thickness in newborns [11]. Therefore, longitudinal assessment of FSCTT has become an important role to identify nutritional fetal abnormalities [10]. However, a longitudinal evaluation of FSCTT in pregnancies after bariatric surgery has not been assessed so far.

Therefore, this study aims to investigate the trajectories of abdominal FSCTT of fetuses conceived after gastric bypass surgery as compared to body mass index (BMI)-matched and obese controls in the second and third trimester of pregnancy. Moreover, their association with maternal glucose metabolism and BMI, as well as time and weight loss after bariatric surgery, should be assessed as further objectives.

\section{Material and Methods}

\section{Research Design}

Details of the study design are reported elsewhere [8]. In short, we retrospectively included 82 singleton pregnancies, 41 women after gastric bypass surgery (GB) and 41 BMI and agematched controls (BMIM), treated at our department between January 2007 and January 2016. Of a total of 86 pregnancies included in the original study, four patients were excluded due to missing ultrasound pictures. In addition, we included 64 obese women with pregestational BMI $\geq 30 \mathrm{~kg} / \mathrm{m}^{2}$ but without history of bariatric surgery $(\mathrm{OB})$. Specialized ultrasound technicians performed all ultrasound examinations with commercially available real-time equipment using standard 3.75-MHz linear or sector transducers transabdominally (Power Vision and Aplio MX, Toshiba, Japan). Longitudinal assessment of FSCTT was performed in a retrospective setting from the second trimester onwards. Thereby, the abdominal FSCTT was assessed after the image was recalibrated: The abdominal circumference level (AC) has been used for all measurements since it is a standardized cutting plane, for unification and therefore having a continuity and equality within the measurements (Fig. 1a). The measure points for the abdominal FSCTT were on the anterior abdominal wall in front of the intrahepatic tract of the umbilical vein, on the left side next to the stomach and on the exact opposite right side of the abdomen. FSCTT was measured in millimeters $(\mathrm{mm})$ between the outer and inner margin of the echogenic subcutaneous tissue after magnifying the area of interest (Fig. 1b). Additionally, medical records were reviewed to collect data on maternal variables such as BMI before and after bariatric surgery and time between operation and pregnancy. Furthermore, results of the $2 \mathrm{~h} 75$ g OGTT including fasting and $60 \mathrm{~min}$ and $120 \mathrm{~min}$ post-load glucose levels (available in $37 \mathrm{~GB}, 33$ BMIM and 64 obese pregnancies), as well as pregnancy outcome and weight of the newborns, were collected. Postprandial hypoglycemia was defined as glucose levels below $70 \mathrm{mg} / \mathrm{dl}$ (alert value) and below $54 \mathrm{mg} / \mathrm{dl}$ (clinically significant biochemical hypoglycemia)
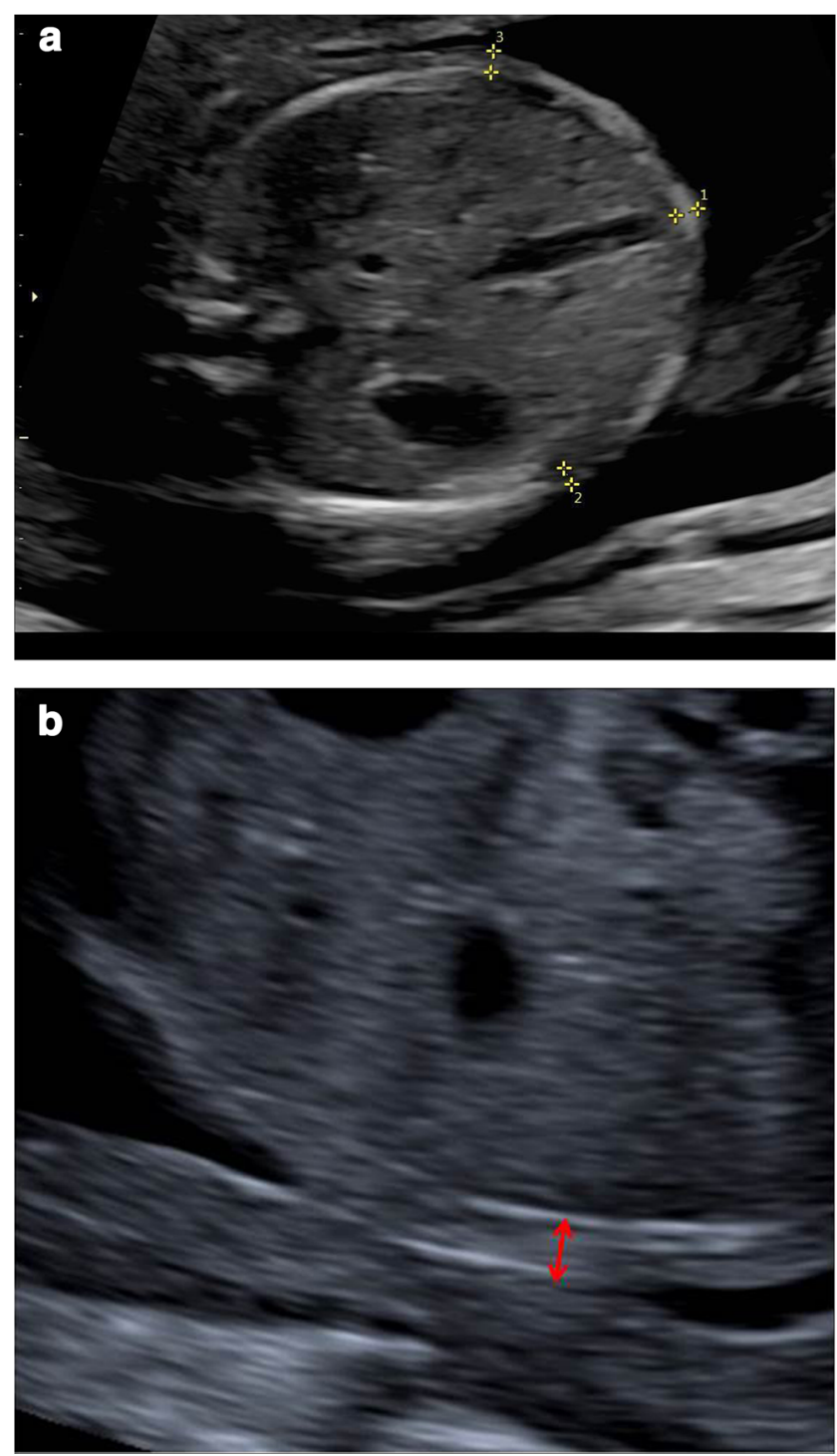

Fig. 1 a An example of the measurement technique of the abdominal fat content at three different points in $20+2$ weeks of gestation. The abdominal circumference level (AC) has been used for all measurements. b The exact measurement of the abdominal FSCTT after magnifying the picture

according to international guidelines [12]. Ethical approval for this study was provided by the Ethics Committee of the Medical University Vienna.

\section{Statistical Analysis}

Continuous variables were summarized by mean \pm standard deviation or median and interquartile ranges (IQR) and compared by analysis of variance (ANOVA) followed by Fisher LSD tests or rank-based inference (Kruskal-Wallis test), respectively. Categorical variables were summarized by counts and percentages and compared by Fisher's exact test. Linear mixed effects (LME) models were used to account for correlated residuals due to repeated measurements. A group by 
time interaction term was included into the LME models to evaluate differences in time-dependent changes between the groups. Statistical analysis was performed with R (V3.5.1) and contributing packages. A two-sided $p$ value $\leq 0.05$ was considered statistically significant.

\section{Results}

Characteristics of the study sample are shown in Table 1 . The median time after GB surgery was 3 years, whereby women after GB showed a mean weight loss of $42.8 \mathrm{~kg}$ between surgery and index pregnancy. Moreover, a high amount postprandial hypoglycemia during the OGTT was observed in this group. FSCTT was significantly lower in fetuses of women after GB as compared to BMI-matched controls in the second (mean difference $1.38 \mathrm{~mm}, 95 \% \mathrm{CI} 1.11-1.65 \mathrm{~mm}, p<0.001$ ) and third trimester of gestation (mean difference $3.37 \mathrm{~mm}$, 95\% CI $2.90-3.83 \mathrm{~mm}, p<0.001)$. Longitudinal analysis revealed significant differences in mean FSCTT trajectories between the groups: FSCTT in offspring of GB mothers increased by $0.37 \mathrm{~mm}$ per 8 weeks of gestation, whereas FSCTT in offspring of BMI-matched controls increased by $2.08 \mathrm{~mm}$ resulting in a significant group per time interaction ( $b=1.71 \mathrm{~mm}, 95 \%$ CI $1.39-2.03 \mathrm{~mm}, p<0.001$, Fig. $2 \mathrm{a})$.
Comparable results were observed when FSCTT in GB offspring were compared to obese mothers who increased by $2.93 \mathrm{~mm}$ (group per time interaction $b=2.56 \mathrm{~mm}, 95 \% \mathrm{CI}$ $2.28-2.85, p<0.001$, Fig. 2a). As visualized in Fig. 2 b, the ratio of FSCTT and abdominal circumference remained constant in the BMI-matched control group and increased with gestational age in fetuses of obese mothers, whereas it significantly decreased in fetuses of women after GB. Further analyses in the GB subgroup found no indication that FSCTT trajectories during pregnancy are notably influenced by OGTT-G mean $(p=0.363)$ or 120 min values $(p=0.651)$, biochemically hypoglycemia below $54 \mathrm{mg} / \mathrm{dl}(p=0.562)$, time since bariatric surgery $(p=0.900)$, or weight loss since surgery $(p=0.852)$. Trajectories of some subgroups are visualized in the supplemental material (Fig. S1-S3).

\section{Discussion}

This study aimed to examine the impact of maternal history of bariatric surgery on fetal development by longitudinal assessment of fetal subcutaneous adipose tissue thickness. As a keyfinding, we observed that fetuses conceived after maternal GB showed markedly reduced ultrasound-derived FSCTT as compared to BMI-matched or obese controls. Moreover, the ratio
Table 1 Characteristics of the study population. Data are mean standard deviation, median (interquartile range) as well as counts (\%); BMI, body mass index; Weight, weight loss since bariatric surgery; time, time between bariatricsurgery and pregnancy; OGTT-G, Glucose during the oral glucose tolerance test; GB, mothers with history of gastric bypass surgery; BMIM, BMI matched controls; $\mathrm{OB}$, obese controls

\begin{tabular}{|c|c|c|c|c|}
\hline & GB & BMIM & OB & $p$ \\
\hline Age (years) & $32.2 \pm 6.3$ & $31.5 \pm 6.0$ & $31.9 \pm 5.9$ & 0.853 \\
\hline Parity & $2(1-2)$ & $2(1-2)$ & $1(0-2)^{*}$ & 0.026 \\
\hline Family history with diabetes & $17(41.5 \%)$ & $14(34.1 \%)$ & $20(31.3 \%)$ & 0.559 \\
\hline History of GDM & $7(17.1 \%)$ & $9(22.0 \%)$ & $8(12.5 \%)$ & 0.440 \\
\hline History of GDM (insulin) & $4(9.8 \%)$ & $6(14.6 \%)$ & $3(4.7 \%)$ & 0.212 \\
\hline Fetal sex (female) & $18(43.9 \%)$ & $21(51.1 \%)$ & $30(47.6 \%)$ & 0.803 \\
\hline Hypothyroidism & $9(22.0 \%)$ & $3(7.3 \%)$ & $7(10.9 \%)$ & 0.116 \\
\hline Mean UA-PI > 1.5 & $1(2.5 \%)$ & $4(10.3)$ & $5(8.6 \%)$ & 0.365 \\
\hline Hypertension or PE & $1(2.4 \%)$ & $1(2.5 \%)$ & $7(10.9 \%)$ & 0.111 \\
\hline Insulin treatment & $7(17.1 \%)$ & $13(31.7 \%)$ & $15(23.4 \%)$ & 0.297 \\
\hline BMI actual $\left(\mathrm{kg} / \mathrm{m}^{2}\right)$ & $29.5 \pm 4.8$ & $29.6 \pm 4.4$ & $35.4 \pm 5.1^{*}$ & $<0.001$ \\
\hline BMI before surgery $\left(\mathrm{kg} / \mathrm{m}^{2}\right)$ & $45.4 \pm 6.9$ & - & - & - \\
\hline$\Delta$ Weight $(\mathrm{kg})$ & $42.8 \pm 14.6$ & - & - & - \\
\hline$\Delta$ time (years) & $3(2-5)$ & - & - & - \\
\hline$\Delta$ time $>1$ (years) & $38(92.6)$ & - & - & - \\
\hline$\Delta$ time $>2$ (years) & $24(58.5)$ & - & - & - \\
\hline OGTT-G 0 min (mg/dl) & $74.4 \pm 6.8$ & $86.2 \pm 7.7^{*}$ & $89.6 \pm 11.4^{*}$ & $<0.001$ \\
\hline OGTT-G 60 min (mg/dl) & $161.8 \pm 44.0$ & $153.7 \pm 32.3$ & $163.0 \pm 37.3$ & 0.501 \\
\hline OGTT-G 120 min (mg/dl) & $67.3 \pm 28.3$ & $114.2 \pm 28.8^{*}$ & $127.2 \pm 32.3 *$ & $<0.001$ \\
\hline OGTT-G mean (mg/dl) & $101.2 \pm 19.1$ & $118.0 \pm 18.1^{*}$ & $126.6 \pm 23.3 *$ & $<0.001$ \\
\hline OGTT-G $120 \leq 70 \mathrm{mg} / \mathrm{dl}$ & $26(70.3 \%)$ & $1(3.0 \%)^{*}$ & $0(0.0 \%)^{*}$ & $<0.001$ \\
\hline OGTT-G $120 \leq 54 \mathrm{mg} / \mathrm{dl}$ & $14(37.8 \%)$ & $0(0.0 \%)^{*}$ & $0(0.0 \%)^{*}$ & $<0.001$ \\
\hline
\end{tabular}



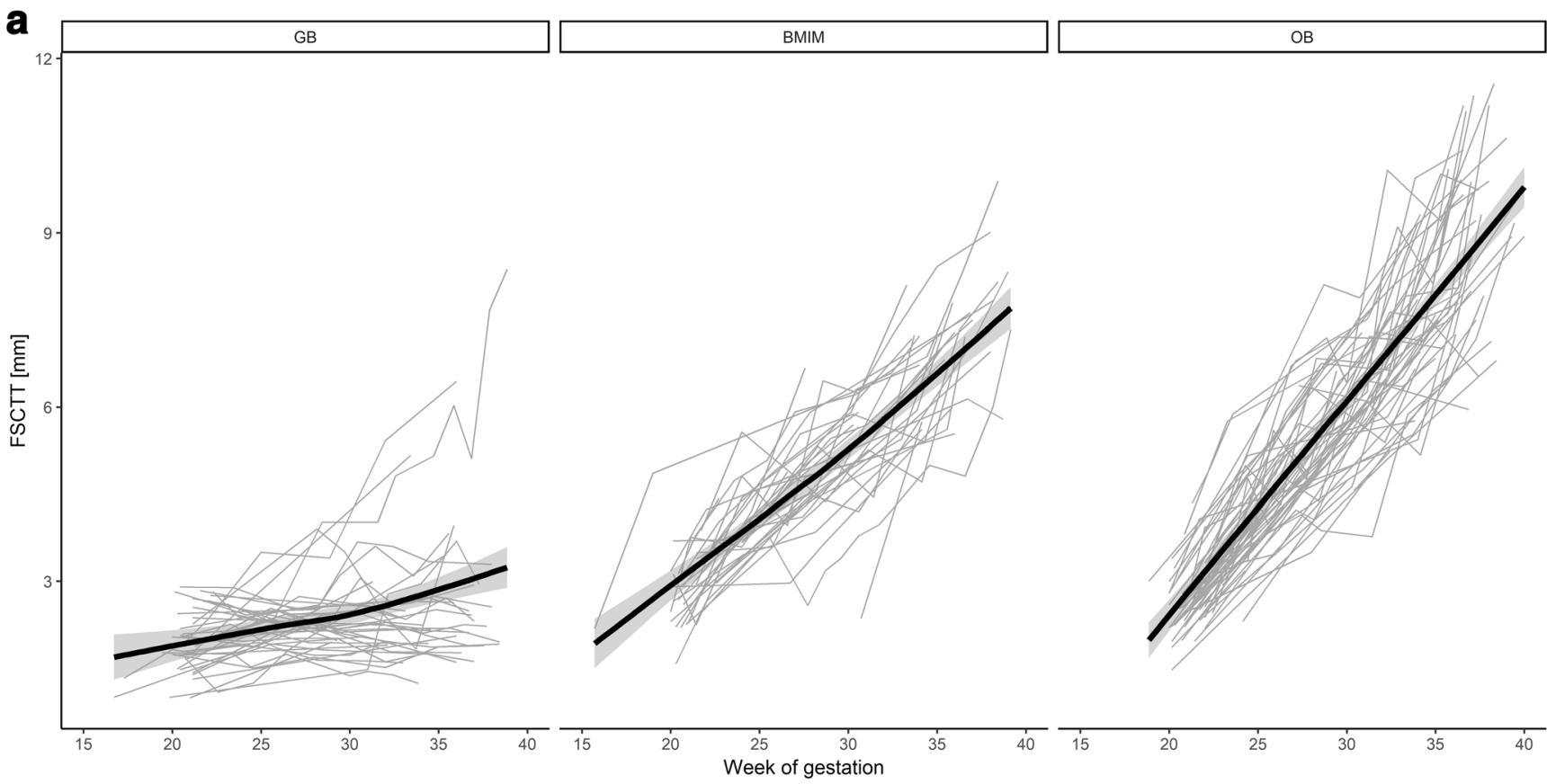

b
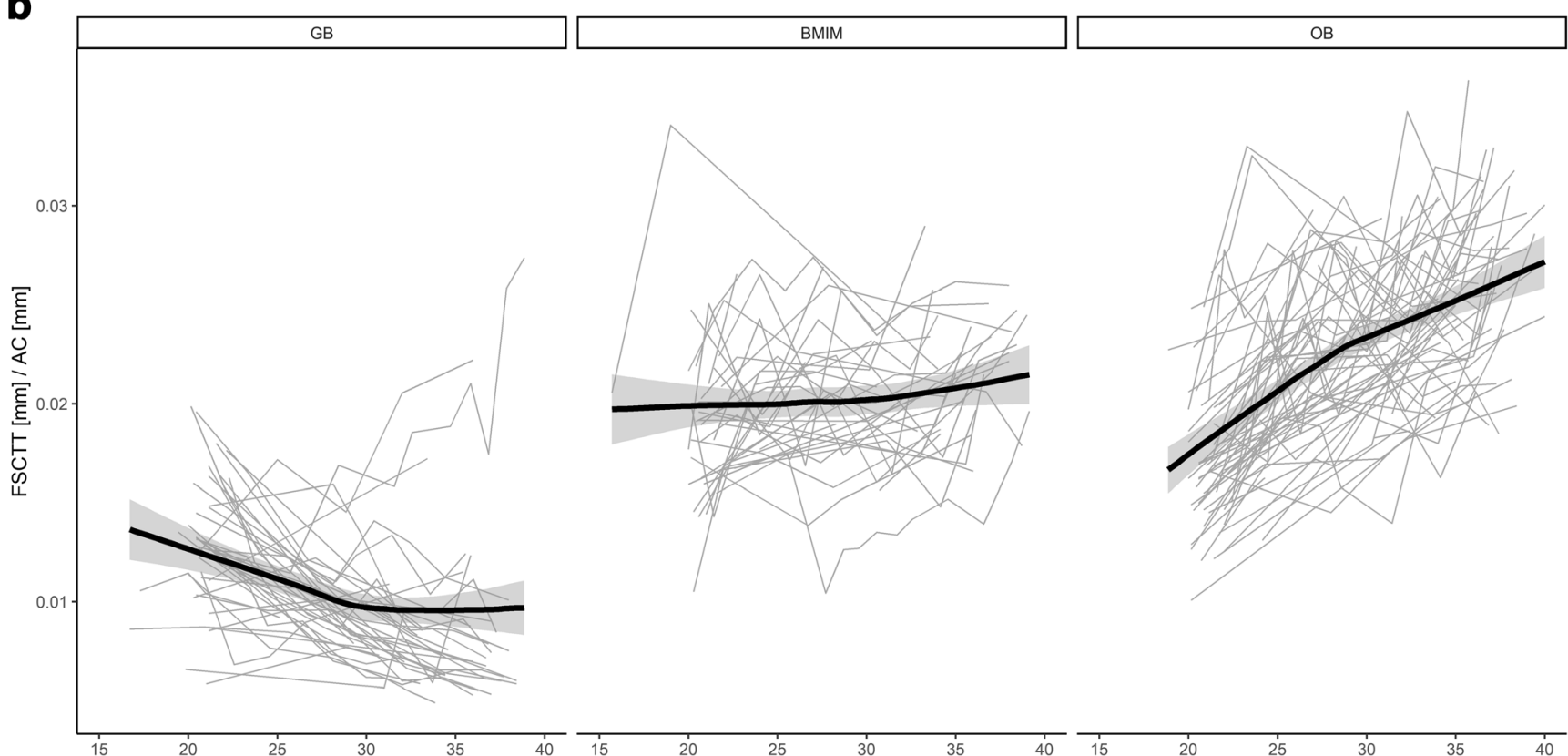

Fig. 2 a Trajectories of fetal abdominal subcutaneous tissue thickness (FSCTT); b Trajectories of FSCTT and abdominal circumference (AC) ratio in pregnancies after gastric bypass (GB) and BMI-matched (BMIM)

of FSCTT and abdominal circumference was markedly decreased in offspring of mothers after GB surgery.

The association between GB and SGA fetuses has been previously reported and discussed $[4,6]$. In addition to these observations, we recently observed markedly decreasing growth curves from the beginning of the second trimester until the end of pregnancy with the largest effect on the abdominal circumference [8]. While the mechanisms beyond these findings are not clarified yet, we assume that development of SGA offspring may be related to metabolic as well as micronutrient deficiencies, which commonly occurred in women after bariatric surgery and especially during pregnancy $[6,13]$. In this regard, some authors hypothesized that the chronically undernourished body of a pregnant women tries to protect itself against nutrient loss through adaptive changes in the placenta, and therefore subsequently causing fetal growth delay [14]. Detailed assessment of fat distribution in offspring of bariatric patients could be helpful to gain a more comprehensive 
understanding of fetal development in pregnancy after weight loss surgery: In the past, several studies found that prenatally reduced abdominal adipose tissue thickness is associated with low birthweight and decreased skinfold thickness [15-18], and hence predictive for impaired nutrition status of the newborns [11]. Moreover, increased fetal subcutaneous fat tissue is known to be a consequence of maternal diabetes and was shown to be more closely related with poor glycemic control than neonatal birth weight [16], suggesting that alterations in maternal glucose metabolism after bariatric surgery contribute to our observation of altered fetal subcutaneous fat trajectories. This is also supported by another recent study on women after GB indicating that birthweight percentiles are related to maternal hyperinsulinemia and glucose nadir levels during a 3-h 75 g OGTT [19]. However, although postprandial hypoglycemia during a routinely performed $2 \mathrm{~h}$ OGTT was frequently observed in the present study, we failed to identify a clear association with reduced FSCTT. While a causal relationship between glycemic alterations and fetal development is obvious, we suggest that more detailed longitudinal examinations of glucose metabolism during pregnancy after GB would be essential to clarify this topic. Only one recent study using continuous subcutaneous glucose monitoring (CGM) in pregnant women with history of GB reported elevated postprandial glucose peaks followed by a rapid fall until $120 \mathrm{~min}$ after ingestion, suggesting considerable high amount of glucose variability in real-life conditions [20]. However, a detailed analysis of possible associations between glucose metabolism and fetal development was not provided in this study.

Some studies observed that time to pregnancy after bariatric surgery, as well as pre-pregnancy weight loss, was associated with perinatal complications [21]. Therefore, several authors recommended to delay pregnancy for at least 12 to 18 month after bariatric operation as pregnancy within this catabolic time frame might lead to altered nutritional supply, and therefore triggering the development of SGA infants [22, 23]. In contrast, others found no differences in neonatal birth weight and adverse pregnancy outcomes between women who conceived within the first postoperative year and those who conceived later [24]. This is also corroborated by our study indicating that reduced FSCTT in offspring of GB mothers was independent of pre-pregnancy weight loss and time since bariatric surgery.

While the association between maternal history of GB and reduced abdominal adipose tissue was remarkable in our study, we were not able to identify a simple explanation for this observation. Although some possible contributors (like maternal serum lipids) are not accessible due to the retrospective study design, we assume a multifactorial genesis with altered metabolism as the most important factor. This applies to women who suffer from other diseases as well: Due to chronic exposure of malnutrition [25, 26], women with eating disorder are at risk for fetal growth delay, comparable to our observation in women after bariatric surgery. Fetal malnutrition and growth delay (whether caused by bariatric surgery, eating disorder or other causes) is assumed to be associated with health consequences in the offspring's later life possibly due to an epigenetic response to fetal nutrition deficiency [27, $28]$ indicating need for further research and long-term observations in this population.

In summary, we observed markedly reduces abdominal adipose tissue thickness in fetuses of mothers with history of bariatric surgery. While the underlying principles for this observation are not fully understood, a multifactorial genesis including nutritional deficiencies and altered metabolism after bariatric surgery may be the most suitable explanation. Further research is warranted to follow up infants of mothers with history of bariatric surgery to assess possible long-term health effect.

Acknowledgements Open access funding provided by Medical University of Vienna.

\section{Compliance with Ethical Standards}

Conflict of Interest The authors declare that they have no conflicts of interest.

Ethical Approval For this type of study, formal consent is not required.

Open Access This article is distributed under the terms of the Creative Commons Attribution 4.0 International License (http:// creativecommons.org/licenses/by/4.0/), which permits unrestricted use, distribution, and reproduction in any medium, provided you give appropriate credit to the original author(s) and the source, provide a link to the Creative Commons license, and indicate if changes were made.

\section{References}

1. Edison E, Whyte M, van Vlymen J, et al. Bariatric surgery in obese women of reproductive age improves conditions that underlie fertility and pregnancy outcomes: retrospective cohort study of UK National Bariatric Surgery Registry (NBSR). Obes Surg. 2016;26: $2837-42$.

2. Mingrone G, Panunzi S, De Gaetano A, et al. Bariatric surgery versus conventional medical therapy for type 2 diabetes. $\mathrm{N}$ Engl $\mathrm{J}$ Med. 2012;366:1577-85.

3. Schauer PR, Bhatt DL, Kirwan JP, et al. Bariatric surgery versus intensive medical therapy for diabetes - 5-year outcomes. N Engl J Med. 2017;376:641-51.

4. Johansson K, Cnattingius S, Näslund I, et al. Outcomes of pregnancy after bariatric surgery. N Engl J Med. 2015;372:814-24.

5. Chevrot A, Kayem G, Coupaye M, et al. Impact of bariatric surgery on fetal growth restriction: experience of a perinatal and bariatric surgery center. Am J Obstet Gynecol. 2016;214:655.e1-7.

6. Kwong W, Tomlinson G, Feig DS. Maternal and neonatal outcomes after bariatric surgery; a systematic review and meta-analysis: do the benefits outweigh the risks? Am J Obstet Gynecol. 2018;218: 573-80.

7. Stephansson O, Johansson K, Näslund I, et al. Bariatric surgery and preterm birth. N Engl J Med. 2016;375:805-6. 
8. Feichtinger M, Falcone V, Schoenleitner T, Stopp T, Husslein PW, Eppel W, et al. Intrauterine fetal growth delay during late pregnancy after maternal gastric bypass surgery. Ultraschall Med. 2018. https://doi.org/10.1055/a-0651-0424.

9. Hammeken LH, Betsagoo R, Jensen AN, et al. Nutrient deficiency and obstetrical outcomes in pregnant women following Roux-en-Y gastric bypass: a retrospective Danish cohort study with a matched comparison group. Eur J Obstet Gynecol Reprod Biol. 2017;216:56-60.

10. Larciprete G, Valensise H, Vasapollo B, et al. Fetal subcutaneous tissue thickness (SCTT) in healthy and gestational diabetic pregnancies. Ultrasound Obstet Gynecol. 2003;22:591-7.

11. Skinner J, O'Donoghue K, Gardeil F, et al. Is fetal abdominal subcutaneous fat comparable with established indices of growth restriction? J Obstet Gynaecol. 2001;21:439-42.

12. International Hypoglycaemia Study Group. Glucose concentrations of less than $3.0 \mathrm{mmol} / \mathrm{L}(54 \mathrm{mg} / \mathrm{dL})$ should be reported in clinical trials: a joint position statement of the American Diabetes Association and the European Association for the Study of Diabetes: Table 1. Diabetes Care. 2017;40:155-7.

13. Coupaye M, Legardeur H, Sami O, Calabrese D, Mandelbrot L, Ledoux S. Impact of Roux-en-Y gastric bypass and sleeve gastrectomy on fetal growth and relationship with maternal nutritional status. Surgery for Obesity and Related Diseases [Internet]. 2018 [cited 2018 Sep 25]; Available from: https://linkinghub.elsevier. com/retrieve/pii/S1550728918304179.

14. Jansson T, Powell TL. Role of placental nutrient sensing in developmental programming. Clin Obstet Gynecol. 2013;56:591-601.

15. Gardeil F, Greene R, Stuart B, et al. Subcutaneous fat in the fetal abdomen as a predictor of growth restriction. Obstet Gynecol. 1999;94:209-12.

16. Tantanasis T, Daniilidis A, Giannoulis C, et al. Sonographic assessment of fetal subcutaneous fat tissue thickness as an indicator of gestational diabetes. Eur J Obstet Gynecol Reprod Biol. 2010;152: 157-62.

17. Aksoy H, Aksoy U, Açmaz G, et al. The effect of impaired 50-gram oral glucose challenge test on fetal abdominal wall thickness. Diabetes Metab Res Rev. 2014;30:570-4.
18. O'Connor C, Doolan A, O'Higgins A, et al. Fetal subcutaneous tissue measurements in pregnancy as a predictor of neonatal total body composition: fetal subcutaneous tissue measurements in pregnancy. Prenat Diagn. 2014;34:952-5.

19. Göbl CS, Bozkurt L, Tura A, et al. Assessment of glucose regulation in pregnancy after gastric bypass surgery. Diabetologia. 2017;60:2504-13.

20. Bonis C, Lorenzini F, Bertrand M, et al. Glucose profiles in pregnant women after a gastric bypass: findings from continuous glucose monitoring. Obes Surg. 2016;26:2150-5.

21. Parent B, Martopullo I, Weiss NS, et al. Bariatric surgery in women of childbearing age, timing between an operation and birth, and associated perinatal complications. JAMA Surg. 2017;152:128.

22. American College of Obstetricians and Gynecologists. ACOG practice bulletin no. 105: bariatric surgery and pregnancy. Obstet Gynecol. 2009;113:1405-13.

23. Busetto L, Dicker D, Azran C, et al. Obesity Management Task Force of the European Association for the study of obesity released "practical recommendations for the post-bariatric surgery medical management.". Obes Surg. 2018;28:2117-21.

24. Kjær MM, Nilas L. Timing of pregnancy after gastric bypass-a national register-based cohort study. Obes Surg. 2013;23:1281-5.

25. Micali N, Stemann Larsen P, Strandberg-Larsen K, et al. Size at birth and preterm birth in women with lifetime eating disorders: a prospective population-based study. BJOG. 2016;123:1301-10.

26. Eik-Nes TT, Horn J, Strohmaier S, et al. Impact of eating disorders on obstetric outcomes in a large clinical sample: a comparison with the HUNT study. Int J Eat Disord. 2018;51:1134-43.

27. Gernand AD, Schulze KJ, Stewart CP, et al. Micronutrient deficiencies in pregnancy worldwide: health effects and prevention. Nat Rev Endocrinol. 2016;12:274-89.

28. Barker DJ. The fetal and infant origins of adult disease. BMJ. 1990;301:1111.

Publisher's Note Springer Nature remains neutral with regard to jurisdictional claims in published maps and institutional affiliations. 